\title{
Modelling of TMF Crack Initiation in Smooth Single-Crystal Superalloy Specimens
}

\author{
Daniel Leidermark, Mikael Segersäll, Johan Moverare and Kjell Simonsson
}

\section{Linköping University Post Print}

\section{Tweet}

N.B.: When citing this work, cite the original article.

Original Publication:

Daniel Leidermark, Mikael Segersäll, Johan Moverare and Kjell Simonsson, Modelling of TMF Crack Initiation in Smooth Single-Crystal Superalloy Specimens, 2014, 11th International Fatigue Congress (Fatigue 2014), 2-7 Mars 2014, Melbourne, Australien.

http://dx.doi.org/10.4028/www.scientific.net/AMR.891-892.1283

Copyright: Not Found

Publisher URL Missing

Postprint available at: Linköping University Electronic Press

http://urn.kb.se/resolve?urn=urn:nbn:se:liu:diva-105336 


\title{
Modelling of TMF crack initiation in smooth single-crystal superalloy specimens
}

\author{
Daniel Leidermark ${ }^{1, \mathrm{a}}$, Mikael Segersäll ${ }^{1, \mathrm{~b}}$, Johan Moverare ${ }^{1,2, \mathrm{c}}$, \\ and Kjell Simonsson ${ }^{1, \mathrm{~d}}$ \\ ${ }^{1}$ Department of Management and Engineering, Linköping University, Sweden \\ ${ }^{2}$ Siemens Industrial Turbomachinery AB, Finspång, Sweden \\ adaniel.leidermark@liu.se, bmikael.segersall@liu.se, cjohan.moverare@liu.se, \\ dkjell.simonsson@liu.se
}

Keywords: Single-crystal superalloy, thermomechanical fatigue, crack initiation, stress relaxation, creep, anisotropy, tension/compression asymmetry.

\begin{abstract}
In this paper the TMF crack initiation behaviour of the single-crystal nickel-base superalloy MD2 is investigated and modelled. TMF tests were performed in both IP and OP for varying mechanical strain ranges in the [001] crystallographic direction until TMF crack initiation was obtained. A crystal plasticity-creep model was used in conjunction with a critical-plane approach, to evaluate the number of cycles to TMF crack initiation. The critical-plane model was evaluated and calibrated at a stable TMF cycle, where the effect of the stress relaxation had attenuated. This calibrated criticalplane model is able to describe the TMF crack initiation, taking tension/compression asymmetry as well as stress relaxation anisotropy into account, with good correlation to the real fatigue behaviour.
\end{abstract}

\section{Introduction}

In the hot sections of gas turbines the components are often made of single-crystal nickel-base superalloys to withstand the high temperatures while still maintaining good mechanical properties, thus achieving a good efficiency of the gas turbine and in turn reducing pollution $[1,2]$. During the startup, steady-state operation and shut-down cycle the temperature and load will vary in the gas turbine, hence the components will be exposed to thermomechanical fatigue (TMF).

Out-of-phase TMF loading conditions were investigated by Amaro et al. [3] for the single-crystal nickel-base superalloy PWA1484. In this paper a mechanism-based crack initiation model was developed and validated. The model takes crystal orientation, applied strain amplitude and stresses, temperature, cycle time and surface roughness into account and a good estimate of the TMF life is achieved. In the work of Moverare et al. [4], where the deformation and damage mechanisms of the single-crystal superalloy CMSX-4 were investigated under TMF loading, it was concluded that the fracture surfaces were entirely crystallographic (parallel to a (111) slip plane), hence indicating that a crack is preferably to initiate and propagate on one of these crystallographic slip planes.

As single-crystal nickel-base superalloys contain an inherent internal structure of discrete slip planes and are deformed in preferred slip directions, it is thus physically motivated that these crystallographic planes may act as potential crack initiation planes. Thus, the critical-plane theory, see e.g. [5], is a useful technique for studying TMF crack initiation in single-crystal material. In the paper by Leidermark et al. [6] a critical-plane model was derived to suit the low-cycle fatigue behaviour of the single-crystal nickel-base superalloy MD2 with good correlation to experimental fatigue crack initiation lives.

The objective of this paper is to define and evaluate a critical-plane model, for describing the TMF crack initiation behaviour of the single-crystal nickel-base superalloy MD2 during both in-phase (IP) and out-of-phase (OP) loading conditions for the [001] crystal orientation. 
Table 1: Experimental test data of the smooth specimens.

\begin{tabular}{r|ccccc}
\hline Specimen & $S 1_{[001]}$ & $S 2_{[001]}$ & $S 3_{[001]}$ & $S 4_{[001]}$ & $S 5_{[001]}$ \\
\hline Diameter [mm] & 6.0 & 6.0 & 6.36 & 6.0 & 6.29 \\
$\Delta \varepsilon_{\text {mech }}[\%]$ & 1.1 & -1.1 & 1.25 & 1.4 & -1.4 \\
$N_{i}$ [cycles] & 1102 & 2158 & 728 & 224 & 156 \\
$\theta\left[^{\circ}\right]$ & 9.4 & 10.2 & 3.3 & 9.5 & 4.5 \\
$\phi\left[^{\circ}\right]$ & 31.1 & 41.3 & 31.3 & 22.1 & 16.5 \\
\hline
\end{tabular}

\section{Experiments}

In this study the nickel-base single-crystal superalloy MD2 with chemical composition Ni-5.1Co6.0Ta-8.0Cr-8.1W-5.0Al-1.3Ti-2.1Mo-0.1Hf-0.1Si in wt. \% was used. The material was first solution heat treated at $1290{ }^{\circ} \mathrm{C}$ for $8 \mathrm{~h}$. Afterwards, a two-stage ageing process with $3 \mathrm{~h}$ at $1100{ }^{\circ} \mathrm{C}$ and $24 \mathrm{~h}$ at $850{ }^{\circ} \mathrm{C}$ was performed. Smooth specimens in the nominal [001] crystal orientation were investigated, where the deviation $(\theta, \phi$, defined as in [7]) from the ideal orientation was determined to be less than $\sim$ $10^{\circ}$ for all specimens. The test specimens were machined from cast bars to a diameter of approximately $6 \mathrm{~mm}$. An Instron servo-hydraulic TMF machine with induction heating and forced cooling was used for the tests. Both IP and OP strain-controlled TMF cycles were applied in which the temperature was cycled between $100-750{ }^{\circ} \mathrm{C}$ with a loading rate of $5{ }^{\circ} \mathrm{C} / \mathrm{s}$, and during which a hold-time of 5 min was applied at maximum temperature, see Fig. 1. Five test specimens were tested at different mechanical strain ranges $\left(\Delta \varepsilon_{m e c h}\right)$, two in both IP and OP loading and the last one in only IP loading, where an extensometer with gauge length of $12.5 \mathrm{~mm}$ was used. All specimens were cycled until a $60 \%$ global load-drop was obtained (defining failure). The number of cycles to TMF crack initiation $\left(N_{i}\right)$ was then determined by a $2 \%$ load-drop of the maximum tensile stress obtained in each cycle, see Table 1 for the experimental data and Fig. 2 for the stress responses of the specimens.

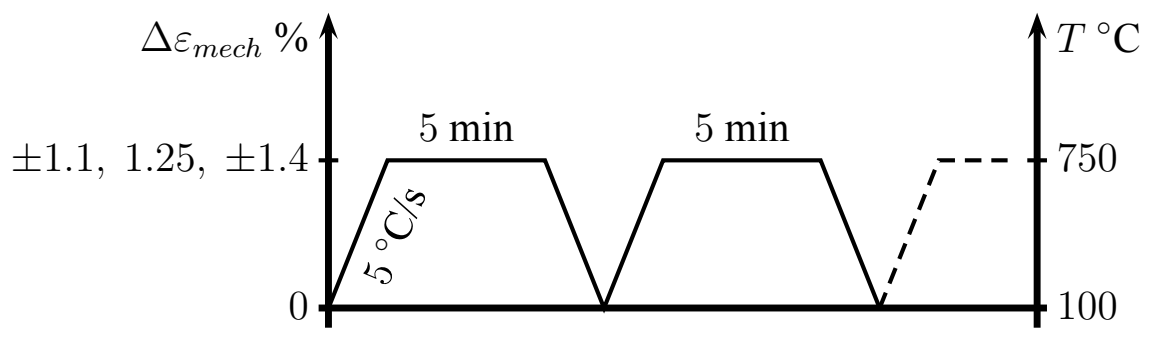

Fig. 1: The TMF cycle which were used in the study.

\section{Modelling}

Constitutive Material Model. The crystal plasticity model developed in Leidermark et al. [7], with the later implemented creep behaviour introduced in Leidermark et al. [8], have been used in all the finite element (FE) simulations. The model is based on crystal plasticity and takes Schmid as well as non-Schmid stresses, elastic anisotropy, tension/compression asymmetry and the anisotropic stress relaxation into account. The inelastic deformation is due to plasticity and creep, thus, the inelastic shear strain rate on the crystallographic slip systems, $\alpha=1, \ldots, 12$, is additively split into a plastic part and a creep part accordingly to

$$
\dot{\gamma}_{i n}^{\alpha}=\dot{\gamma}_{p}^{\alpha}+\dot{\gamma}_{c}^{\alpha}
$$

The yield functions in plasticity for the slip systems are given by

$$
f^{\alpha}=\sigma_{e}^{\alpha}-G_{r}
$$



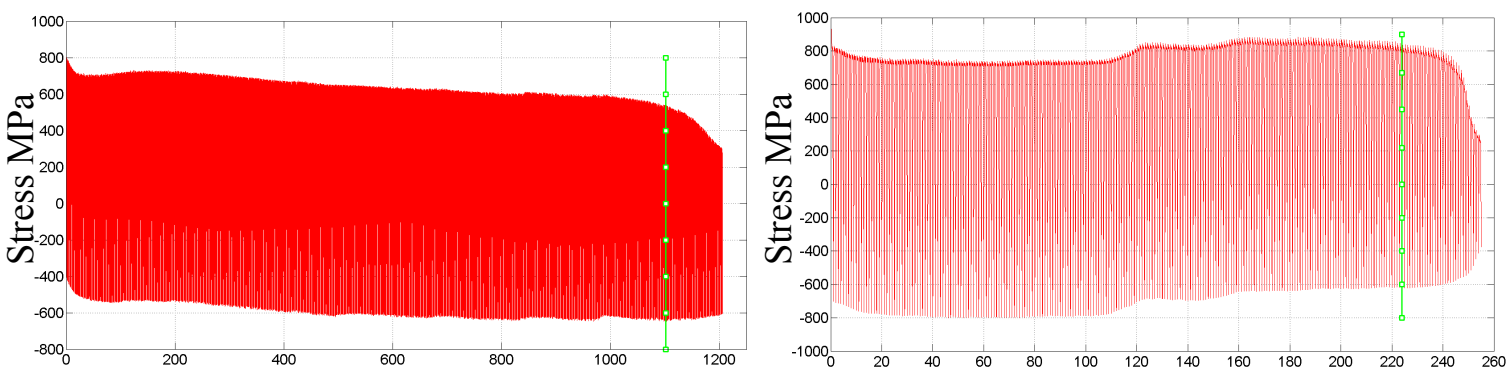

a) $S 1_{[001]}$

b) $S 4_{[001]}$

$N_{i}$
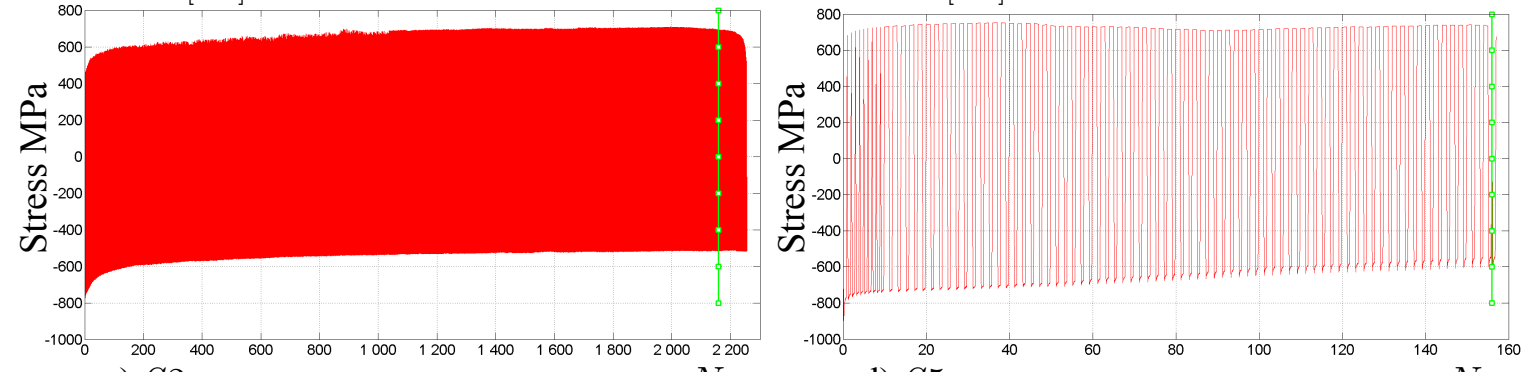

c) $S 2_{[001]}$

$N_{i}$

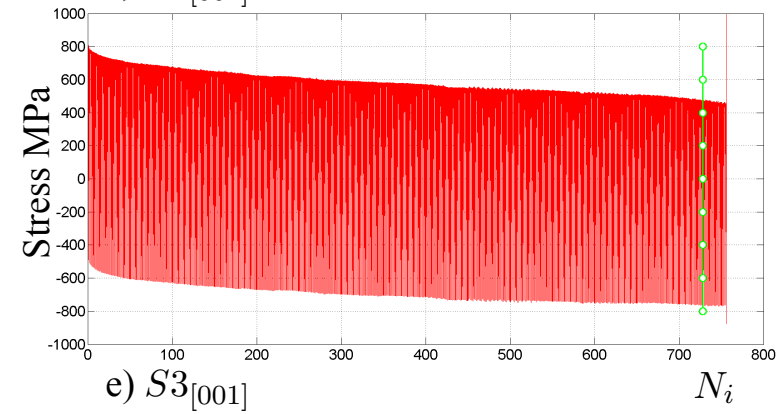

d) $S 5[001]$

$N_{i}$

Fig. 2: The experimental TMF response of specimen a) $S 1_{[001]}$, b) $S 4_{[001]}$, c) $S 2_{[001]}$, d) $S 5_{[001]}$ and $S 3_{[001]}$. The green (dotted) line corresponds to the number of cycles to TMF crack initiation ( $2 \%$ loaddrop).

where $G_{r}$ is the slip resistance on the plane, and has the same value for all slip systems, and the following macroscopic equivalent stresses were employed

$$
\sigma_{e}^{\alpha}=\left|\tau_{p b}^{\alpha}\right|+\kappa_{1}\left|\tau_{c b}^{\alpha}\right|+\kappa_{2}\left|\tau_{s b}^{\alpha}\right|+\kappa_{3} \tau_{p e}^{\alpha}+\kappa_{4} \tau_{s e}^{\alpha}+\kappa_{5} \sigma_{p n}^{\alpha}
$$

where the first shear stress $\tau_{p b}^{\alpha}$ is the Schmid stress and the following five are non-Schmid stresses, and the $\kappa_{1}$ through $\kappa_{5}$ are weight factors that describe how the non-Schmid stresses affect the yield limit. The weight factors and $G_{r}$ are temperature dependent parameters, which are determined from experimentally obtained yield data. The first three terms in Eq. (3) set the magnitude of the yield surfaces, while the asymmetry in tension and compression is described by the three last terms. It is to be noted that the Schmid and non-Schmid stresses, in a modelling context, represent mean value stresses over a matrix with ordered $\gamma^{\prime}$-particles. Finally, the following non-associated flow rule was adopted

$$
\dot{\gamma}_{p}^{\alpha}=\dot{\lambda}_{p}^{\alpha} \frac{\partial g^{\alpha}}{\partial \tau_{p b}^{\alpha}}=\dot{\lambda}_{p}^{\alpha} \operatorname{sgn}\left(\tau_{p b}^{\alpha}\right)
$$

where the non-associated flow is in the direction of the Burger's vector $g^{\alpha}=\left|\tau_{p b}^{\alpha}\right|$, and in order to handle rate independent crystal plasticity, the following viscoplastic flow function was used

$$
\dot{\lambda}_{p}^{\alpha}=\dot{\gamma}_{0}\left\langle\left(\frac{\sigma_{e}^{\alpha}}{G_{r}}\right)^{m}-1\right\rangle
$$

where $\dot{\gamma}_{0}$ and $m$ are regularization parameters, which were given the values 0.1 and 10 . 
To be able to handle the stress relaxation during the hold-times, a creep law based on the basic Norton creep law was adopted for each slip system, see Leidermark et al. [8]. A non-associated flow rule for creep was also defined in the direction of the shear stress $\tau_{p b}^{\alpha}$, as in the flow rule for plasticity, and was thus given by

$$
\dot{\gamma}_{c}^{\alpha}=\dot{\lambda}_{c}^{\alpha} \frac{\partial g^{\alpha}}{\partial \tau_{p b}^{\alpha}}=\dot{\lambda}_{c}^{\alpha} \operatorname{sgn}\left(\tau_{p b}^{\alpha}\right)
$$

where the magnitude is defined accordingly to

$$
\dot{\lambda}_{c}^{\alpha}=C(\boldsymbol{S})\left(\frac{\sigma_{e}^{\alpha}}{G_{r}}\right)^{n} A(T)
$$

in which the creep exponent $n$ is set to 12.0087 , and where $C(\boldsymbol{S})$ is the anisotropic creep coefficient used to register the type of crystallographic loading. In detail

$$
C(\boldsymbol{S})=b_{0}+b_{1} x_{1}(\boldsymbol{S})+b_{2} x_{2}(\boldsymbol{S})
$$

where $\boldsymbol{S}$ is the second Piola-Kirchhoff stress tensor, and where $b_{0}, b_{1}$ and $b_{2}$ are linear regression parameters with the values of $-0.00224667,-0.022414$ and 0.040254 respectively, these values and $n$ were obtained by a parameter optimisation procedure, and where the $x_{1}(\boldsymbol{S})$ and $x_{2}(\boldsymbol{S})$ are the maximum values obtained by projecting the second Piola-Kirchhoff stress tensor on the crystal orientations of the $\langle 011\rangle$ and $\langle 111\rangle$ families, i.e.

$$
\left\{\begin{array}{l}
x_{1}(\boldsymbol{S})=\max \left(\langle 011\rangle \boldsymbol{S}\langle 011\rangle^{T}\right) \\
x_{2}(\boldsymbol{S})=\max \left(\langle 111\rangle \boldsymbol{S}\langle 111\rangle^{T}\right)
\end{array}\right.
$$

Finally, in Eq. (7), $A(T)$ is the Arrhenius factor which accounts for the thermal activation of the creep process and is defined as

$$
A(T)=A_{0} \mathrm{e}^{-\frac{Q}{R T}}
$$

where the parameters are the universal gas constant $R=8.3144621 \mathrm{~J} /(\mathrm{mol} \mathrm{K})$, the activation energy $Q=264730 \mathrm{~J} / \mathrm{mol}$, a constant $A_{0}=3.273 \cdot 10^{13}$ and the temperature $T$ in $\mathrm{K}$.

Critical-Plane Model. The critical-plane model defined by Leidermark et al. [6] has been adopted for TMF loadings, in which the total shear strain ranges on the crystal planes are investigated for determining the critical slip system, i.e. the crystal slip system which experiences the maximum strain range. When the maximum strain range is found (in one of the elements in the FE-model under consideration), it is used in a life function of Coffin-Manson type to determine the number of cycles to TMF crack initiation, i.e.

$$
\Delta \gamma_{\max }^{t o t}=f\left(N_{i}\right)=p N_{i}^{q}
$$

where the constants $p$ and $q$ are determined from the TMF experiments.

\section{Evaluation and Calibration of TMF Crack Initiation}

FE-simulations were performed of the specimens using the FE-software LS-DYNA [9], version R7.0, with the user-defined crystal plasticity-creep model. Due to computational aspects regarding the userdefined crystal plasticity-creep model and complications in applying the boundary conditions to achieve the correct $\Delta \varepsilon_{\text {mech }}$ in the TMF cycles, only the smooth part over the gauge length of the whole specimens was modelled and analysed. A post-processing operation was made, in which the maximum total shear strain range found on a crystal plane in the elements around the circumference at mid-length of the specimen was extracted from each simulation, see Table 2 for data regarding the extraction. 
Table 2: Extracted data from the FE-simulations

\begin{tabular}{r|ccccc}
\hline Specimen & $S 1_{[001]}$ & $S 2_{[001]}$ & $S 3_{[001]}$ & $S 4_{[001]}$ & $S 5_{[001]}$ \\
\hline$\Delta \gamma_{\max }^{\text {tot }}[\%]$ & 1.3390 & 1.3475 & 1.4541 & 1.7242 & 1.6577 \\
Slip system & 2 & 2 & 2 & 2 & 2 \\
Element & 2360 & 2344 & 649 & 2368 & 2352 \\
\hline Slip system 2 is the crystallographic equivalence of $(111)[\overline{101]}$.
\end{tabular}

During TMF loading the component will undergo stress relaxation as seen in Fig. 2. Since the TMF loading is performed under mechanical strain-control, i.e. the applied strain range is constant, the evaluation of $\Delta \gamma_{\max }^{t o t}$ is performed during the on-loading after the third TMF cycle, where any variations in shear strain range have attenuated and a stable response is received. This procedure is due to the fact that a simulation of all the TMF cycles until crack initiation is too computationally expensive. The response of the four first TMF cycles can be viewed in Fig. 3, where a comparison between the experimental and simulated responses of the specimens is made.

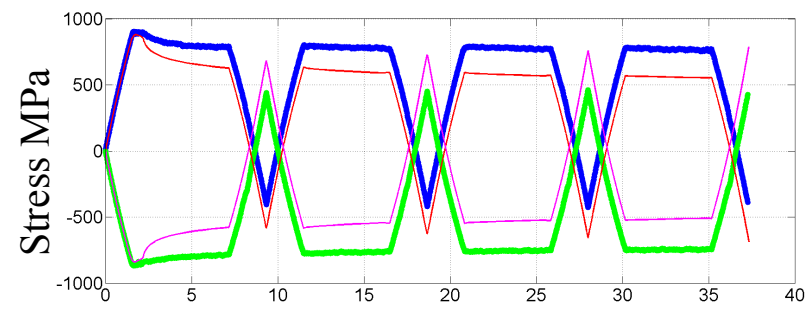

a) $S 1_{[001]}$ and $S 2_{[001]}$

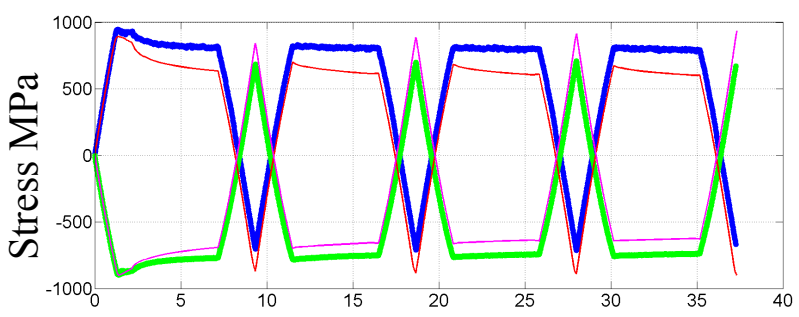

b) $S 4_{[001]}$ and $S 5_{[001]}$

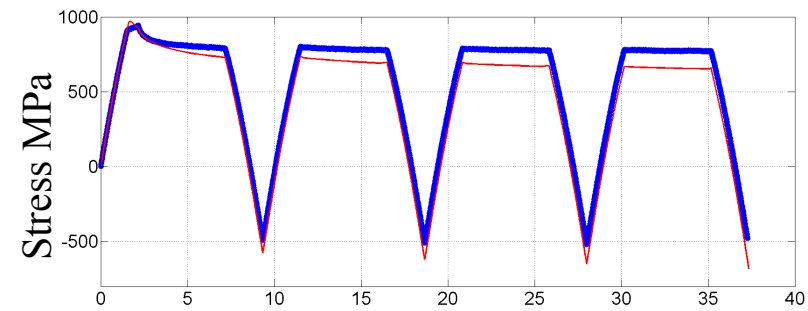

c) $S 3_{[001]}$

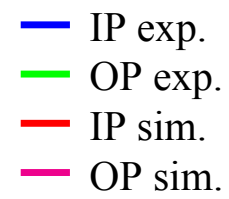

Fig. 3: The stress relaxation of the four first TMF cycles of the experimental and simulated responses of the specimens a) $S 1_{[001]}$ and $S 2_{[001]}$, b) $S 4_{[001]}$ and $S 5_{[001]}$ and c) $S 3_{[001]}$.

The extracted $\Delta \gamma_{\max }^{\text {tot }}$, from the four specimens where both IP and OP loading conditions were performed ( $S 1_{[001]}, S 2_{[001]}, S 4_{[001]}$ and $\left.S 5_{[001]}\right)$, were used to define the critical-plane model. These shear strain ranges were plotted versus the experimental number of cycles to TMF crack initiation and the parameters in the critical-plane model were obtained by a curve-fitting procedure of these points, where the values $p=0.0284$ and $q=-0.1010$ were obtained, see Fig. 4 . The last specimen $\left(S 3_{[001]}\right)$ was used to validate the critical-plane model.

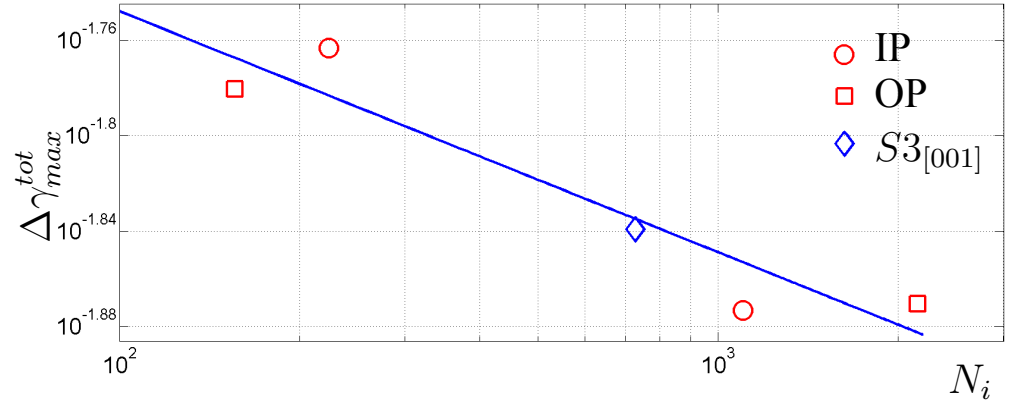

Fig. 4: The received maximum total shear strain range $\left(\Delta \gamma_{\text {max }}^{\text {tot }}\right)$ versus the experimental TMF crack initiation lives plotted in log-log scale with the curvefitted line in blue. 


\section{Validation and Concluding Remarks}

The calibrated critical-plane model is validated by the experimental and simulated response of specimen $S 3_{[001]}$, which was not included in the development of the critical-plane model describing the TMF crack initiation. From the conducted FE-simulation the $\Delta \gamma_{\max }^{\text {tot }}=1.4541 \%$ was received, hence yielding 755 cycles to TMF crack initiation when using the calibrated critical-plane model. Comparing these number of cycles to TMF crack initiation with the experimentally obtained cycles, one sees a small discrepancy with an error of $3.7 \%$.

Hence, on the basis of the presented findings and the validation it is concluded that:

- The TMF behaviour (stress relaxation, anisotropy) observed in the experiments are captured well with the crystal plasticity-creep model used in the FE-simulations.

- A good correlation with the real TMF behaviour is achieved, as can be verified due to the small error $(3.7 \%)$ found in the analysis of $S 3_{[001]}$.

- The tension/compression asymmetry behaviour (IP and OP) is well captured by the critical-plane model.

- Only smooth specimens were addressed in the study, hence no stress concentration effects were taken into account. This need to be done in e.g. notched geometries where, as a consequence, the stress relaxation plays a far more important role.

- Another observation is that the critical slip system in the simulations always remains the same. A reason why this is true might be that the deviation in orientation is small and within the same region for all specimens, and that the analysed FE-model was only a part of the whole specimen.

\section{Acknowledgement}

The work has been financially supported by Siemens Industrial Turbomachinery AB in Finspång, Sweden, and the Swedish Energy Agency, via the Research Consortium of Materials Technology for Thermal Energy Processes, Grant No. KME-502.

\section{References}

[1] R.C. Reed: The Superalloys - Fundamentals and Applications (Cambridge University Press, UK, 2006)

[2] A. Pineau and S.D. Antolovich: Engineering Failure Analysis, Vol. 16, (2009), p. 2668

[3] R.L. Amaro, S.D. Antolovich and R.W. Neu: Fatigue \& Fracture of Engineering Materials \& Structures, Vol. 35, (2012), p. 658

[4] J.J. Moverare, S. Johansson and R.C. Reed: Acta Materialia, Vol. 57, (2009), p. 2266

[5] A. Karolczuk and E. Macha: International Journal of Fracture, Vol. 134, (2005), p. 267

[6] D. Leidermark, J. Moverare, K. Simonsson, S. Sjöström and S. Johansson: Procedia Engineering, Vol. 2, No. 1, (2010), p. 1067

[7] D. Leidermark, J.J. Moverare, K. Simonsson, S. Sjöström and S. Johansson: Computational Materials Science, Vol. 47, No. 2, (2009), p. 366

[8] D. Leidermark, M. Segersäll, J. Moverare and K. Simonsson: submitted for publication (2013)

[9] J.O. Hallquist: LS-DYNA Theory Manual (Livermore Software Technology Corporation, USA, 2006) 